Casos C línicos

Arch. Esp. Urol., 58, 9 (960-963), 2005

\section{HEMANGIOMA CAVERNOSO COMO CAUSA DE OBSTRUCCIÓN URETEROPIÉUCA}

Roberto Ferrero Doria, Francisco G arcía Víctor, Fermín M oreno Pérez, M arcos $G$ assó $M$ a toses y Enrique Díaz Calleja.

Servicio de Urología. Hospital Francisco de Borja de Gandía. Valencia. España.

Resumen.- O BJETIVO: Presentación de un caso de hemangioma cavernoso situado en el tracto urinario superior.

M ÉTO DO / RESULTADO S: M ujer de 29 años con clínica de crisis renoureteral derecha que tras estudio con UIV se objetiva estenosis de la unión pieloureteral derecha. Se realiza una pieloplastia desmembrada y se observa que era un hemangioma cavernoso el causante de dicha estenosis.

CON CLUSIO NES: El hemangioma cavernoso, patología rara en el tracto urinario superior, tiene que ser considerarado como uno de las posibles causas de estenosis de la unión pieloureteral.

Palabras clave: Estenosis pieloureteral. AndersonHynes.
Summary- O BJEC TIVES: To report one case of cavernous haemangioma in the upper urinary tract.

METHODS/ RESULTS: 29-year-old female presenting with right renal colic whose IVP showed UPJ obstruction. Dismembered pyeloplasty was performed and pathology reported a cavernous haemangioma as the cause of stenosis.

CONCLUSIONS: Cavernous haemangioma, a rare upper urinary tract pathology, should be considered in the differential diagnosis of causes of UPJ obstruction.

Keywords: Ureteral pyelic junction obstruction. Anderson Hynes

\section{INTRODUCCIÓN}

El hemangioma es una lesión vascular benigna que raramente se sitúa en el aparato urinario alto. La mayoría de casos descritos son descubiertos tras el estudio de una hematuria total macroscópica unilateral, utilizando mayoritariamente técnicas endoscópicas de la vía urinaria alta.

Por otro lado la estenosis de la unión pieloureteral es una patología relativamente común y más propia de la edad infantil. Múltiples son las causas que la provocan, siendo la más frecuente la presencia de un segmento aperistáltico con alteración de la estructura histológica del mismo. Presentamos un caso de hemangioma cavernoso en el aparato urinario alto, diagnosticado en nuestro Servicio de Urología, como causa de estenosis de la unión pieloureteral.

\section{CASO CĹNICO (M.D.M.C.)}

Mujer de 29 años con antecedentes de ulcus duodenal y estreñimiento que consulta por dolor en fosa renal derecha compatible con crisis renoureteral. $\mathrm{No}$ antecedentes de nefrolitiasis ni hematuria ni infecciones del tracto urinario. En la exploración sólo destaca una puñopercusión renal derecha positiva. La ecografía objetiva ectasia pielocalicial renal derecha con adelgazamiento del parénquima. La UIV muestra una anulación funcional de la unidad renal derecha siendo normal el resto de la exploración. Una pielografía retrograda muestra estenosis en la unión pieloureteral derecha (Figura 1), siendo la citología
Roberto Ferrero Doria

Avda. Albaida 2, escalera 1, piso 6ㅇ, puerta 18. 468700 ntinyent. Valencia. España. e-mail: alenata@inicia.es

Trabajo recibido: 
urinaria selectiva del uréter derecho negativa. A nte la disyuntiva de practicar una cirugía reconstructiva o una exerética se realiza gammagrafía renal que demuestra captación relativa del 33\% para el riñón derecho y del $67 \%$ para el izquierdo.

Se realiza pieloplastia renal derecha según técnica de Anderson-Hynes.

El examen anatomopatológico de la estenosis de la vía urinaria descubre un hemangioma cavernoso (Figura 2) como causante de la obstrucción, el cual no permitía pasar ninguna cánula.

El control postoperatorio urográfico a los 3 meses (Figura 3) objetiva captación y eliminación renal derecha de contraste con ligero retraso respecto a riñón izquierdo.

La paciente fue dada de alta de consultas externas a los tres años de la cirugía tras haber sido practicados otros dos controles urográficos; 7 años después de la pieloplastia no ha vuelto a consultar por clínica urológica.

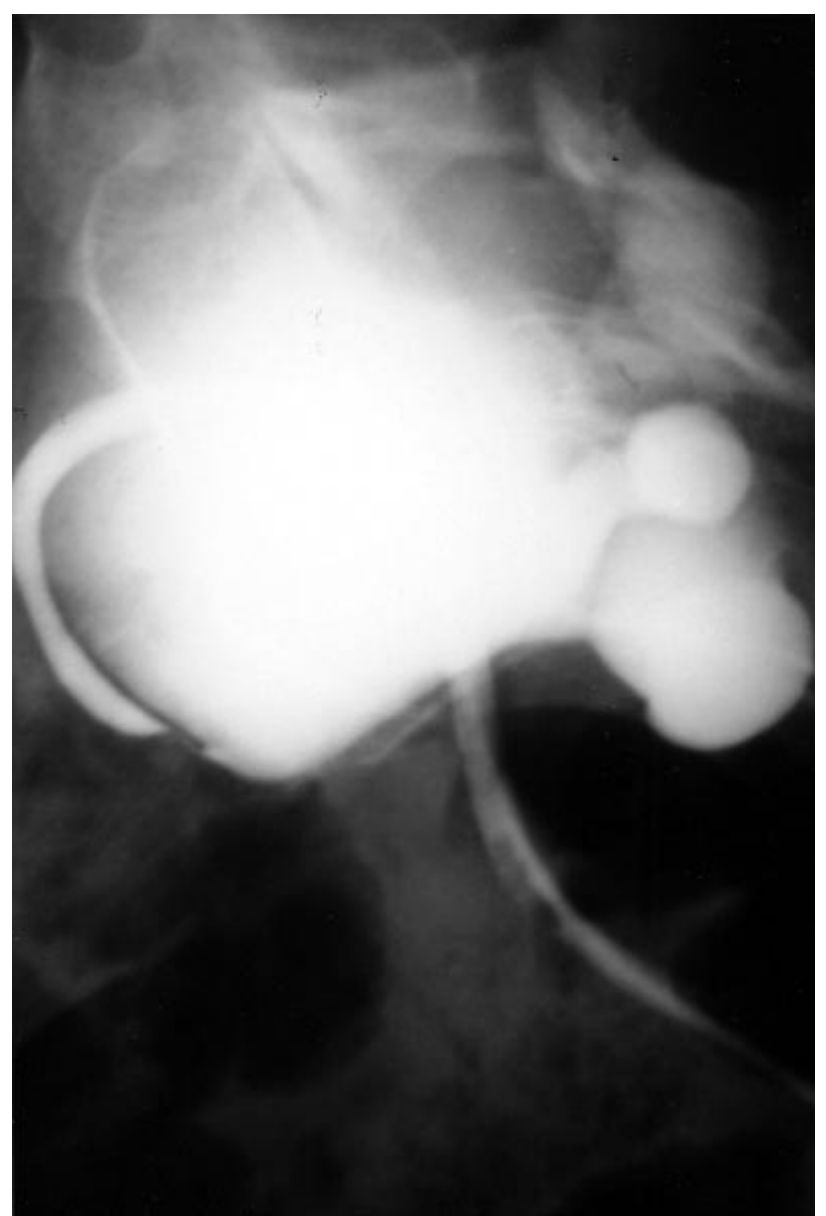

FIG URA 1. Pielografía retrógrada derecha.

\section{DISCUSIÓN}

Los hemangiomas son considerados como tumores benignos sin potencial degeneración maligna (1).

Habitualmente se localizan en la piel, membranas mucosas y en el hígado. Muy raramente se localizan en el riñón. En una revisión antigua, Riley y Swann describen que los mismos, cuando se localizan en el riñón, pueden estar ubicados en la corteza, área medular, papilas y pelvis (2). En una serie descrita por Bagley y Allen y diagnosticados tras estudio de hematuria esencial utilizando ureteroscopio flexible, reflejan que la localización más frecuente de esta lesión, dentro del riñón, es en la papila renal (3).

En algunas ocasiones se presentan como masas renales, aunque en la mayoría de los casos, suele presentarse como pequeñas lesiones $(1,4)$.

La incidencia de estas lesiones suele variar según publicaciones, aunque hemos de recurrir a series de autopsias para averiguar este dato. Peterson y Thompson publicaron en 1971 un artículo que incluía la revisión de 6 series de autopsias, un total de 132.000 casos, encontrando únicamente 4 casos de estos hemangiomas renales (5).

Han sido clasificados en papilares, cavernosos, mixtos y arterio-venosos (6).

La edad de diagnóstico oscila entre los 16 y 40 años. Son pacientes que presentan dolor lumbar y/ o hematuria macroscópica, que obliga a realizar un diagnóstico diferencial con nefrolitiasis, tumores benignos o malignos de riñón, angiomiolipomas, linfangiomas o simple y llanamente una infección del tracto urinario (7).

Raramente el diagnóstico de esta lesión suele ser preoperatorio. Las técnicas habituales para su estudio pasan por la urografía intravenosa, la citología, alteraciones de la coagulación y a menudo el TAC y la angiografía (3). La gammagrafía con hematíes marcados con Tecnecio-99 puede ayudarnos a diagnosticar un hemangioma de la vía urinaria, si éste produce hematuria y se pretende averiguar el origen de la misma, pero son la técnicas endoscópicas, anterógradas o retrógradas, las que realmente nos ayudan al diagnóstico exacto de esta lesión (6). Bien es cierto que la visualización de las lesiones no siempre es diagnóstica y puede ofrecer cierta dificultad en la filiación de las mismas, debiendo distinguir entre neoplasias de alto grado, lesiones inflamatorias, cálculos, etc (3) 


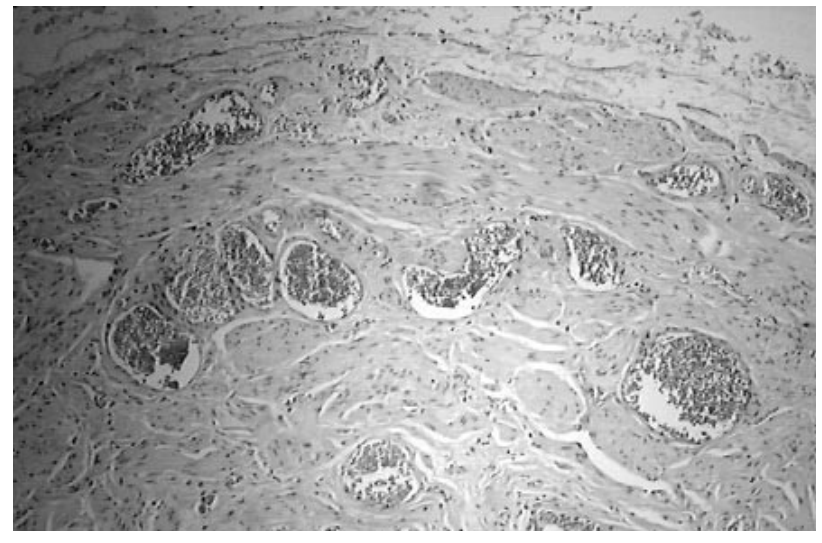

FIG URA 2. Histología del hemangioma.

El tratamiento de esta patología suele ser conservador. Un criterio importante a la hora de decantarse por una u otra opción va a ser el tamaño del hemangioma. Algunas lesiones pueden experimentar una fibroesclerosis de forma espontánea. También puede optarse por la embolización selectiva. Algunos autores propugnan la enucleación del tumor cuando éste es sintomático y de menos de $2 \mathrm{~cm}$, aunque ante la posibilidad de ser multifocal, puede que sea un tratamiento incompleto (7). Está descrita la

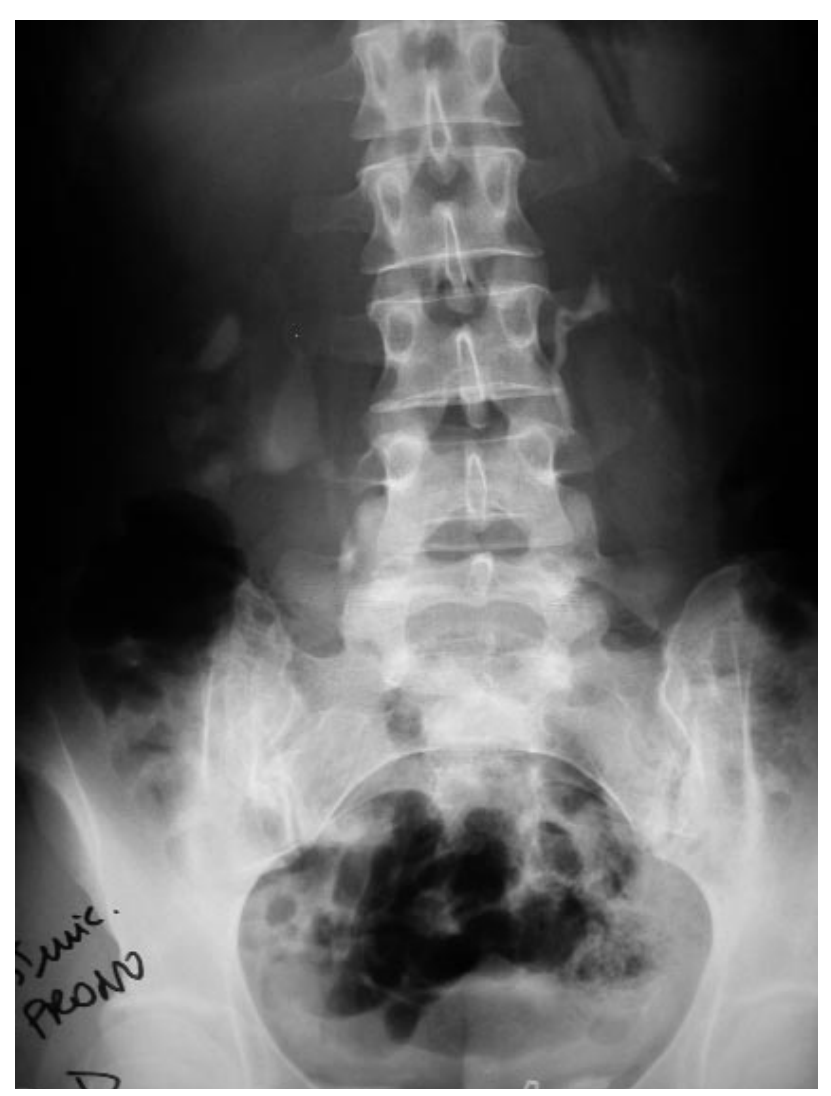

FIG URA 3. UIV tras pieloplastia. posibilidad de tratamiento farmacológico con nitrato de plata al 1\% instilado de forma retrógrada o la administración sistémica de ácido aminocaproico (3). 0 tra opción pasa por la realización de una nefrectomía parcial en aquellos casos con lesiones localizadas. Finalmente, puede considerarse la nefrectomía cuando el diagnóstico no es lo suficientemente claro, ante hemorragias masivas 0 ante enfermedad multifocal $(3,7)$. Las técnicas endourológicas, en su doble vertiente, diagnósticas y terapéuticas, son las más utilizadas, ya que permiten la visualización de la lesión y su posterior fulguración. Los ureteroscopios flexibles son los más resolutivos en estos casos, pues permiten la inspección visual de todas las cavidades intrarrenales, permitiendo la visualización de toda la superficie urinaria intrarrenal en el $95 \%$ de los casos Es necesario una visión muy clara, especialmente si el sangrado está presente, para que la fulguración sea satisfactoria, así como un adecuado canal de trabajo para el paso de instrumental (3).

Por otro lado, la estenosis de la unión pieloureteral es una patología congénita cuya incidencia es de aproximadamente 5/100000. Predomina en el hombre con una relación hombre/mujer de $2 / 1$ a $5 / 2$ y más en el lado izquierdo ( distribución $5 / 2$ ). Bilateral entre el $10-15 \%$ de los casos y asociada a otras anomalías urológicas congénitas en el $50 \%$ de los casos (8).

Los tipos de obstrucción pieloureteral, según una clasificación anatómica, pueden ser por estenosis intrínseca, anomalías de inserción, bandas fibrosas o vasos transversales (9). En las estenosis intrínsecas hay un doble problema: uno físico y otro funcional. Las luces de la unión poseen un revestimiento transicional habitual, pero el número de células musculares que las rodea es menor. En algunos casos hay una hipertrofia muscular anómala en la unión pieloureteral e incluso está descrito un aumento de tejido rico en colágeno. En estos casos se trataría de un segmento aperistáltico. Las estenosis por anomalías de la inserción no están lo suficientemente claras. El riñón es una víscera "móvil", suspendida y anclada en el retroperitoneo, por lo que una dilatación en la pelvis obligaría a que la unión pieloureteral pasara a adoptar una posición relativamente alta. Por ello las anomalías en la inserción no se saben si son el origen o la consecuencia de un problema previo. Así mismo han sido descritos casos de bandas fibrosas que pudieran ser consecuencia de agresiones como nefrostomías, infecciones urinarias,.. que generan una fibrosis periureteral. Finalmente, y dentro de los tipos de obstrucción pieloureteral, se han implicado la presencia de vasos transversales en esta localización anatómica. No siempre implica que estos vasos sean la 
causa de la obstrucción, pero casi todos los autores afirman que el $25 \%$ de los casos de obstrucción pieloureteral productora de hidronefrosis se asocia a una variante ureterovascular. 0 tro dato, que no ayuda precisamente al esclarecimiento de esta causa vascular, es el descrito por Ross (10), quien refiere que en las hidronefrosis de diagnóstico prenatal y que posteriormente fueron intervenidas quirúrgicamente, no suelen existir vasos polares. Dentro de todas la etiologías posibles de obstrucción de la unión pieloureteral, y tras consultar la bibliografía, no se describen casos en que la misma esté causada por un hemangioma. Si hemos encontrado un artículo de asociación de estenosis de la unión pieloureteral con un hemangioma renal (11).

Para finalizar, poner en consideración de los urólogos que ante una estenosis de la unión pieloureteral habrá que considerar la presencia de un hemangioma como posible origen de la misma.

\section{BIBUOGRAFÍA y LECTURAS RECOMENDADAS (*lectura de interés y **lectura fundamental)}

1. AKEL, S.R.; RASSI, A.; TAWL, A. y cols.: "Isolated renal haemangioma in children: presentation and management". BJU Int., 90: 758, 2002.

2. RILEY, A.; SWANN, W.J.Jr.: "Angioma of the kidney". Urol. Cutan. Rev., 45: 377, 1941.

3. BAGLEY, D.H.; ALLEN, J.: "Flexible ureterospyeloscopy in the diagnosis of benign essential hematuria". J. Urol., 143: 549, 1990.

*4. DANESHMAND, S.; HUFFMAN, J.L.: "Endoscopic management of renal hemangioma". J. Urol., 167: 488, 2002.

**5. PETERSON, N.; THOMPSON, H.T.: "Renal hemangioma". J. Urol., 105: 27, 1971.

**6. PÉREZ, M.P.; CAMPOY, P.; CHICHÓN, D. y cols.: "Angiomatosis pélvica como causa de hematuria". Actas Urol. Esp., 28: 462, 2004.

7. MOODY, J.A.; LITWIN, M.S.; COCHRAN, S.T. y cols.: "Renal cavernous hemangioma in a patient with the acquired immunodeficiency syndrome". J. Urol., 156: 1759, 1996.

*8. ROARKE, M.C.; SANDLER, C.M.: "Clínicas de Urología de Norteamérica. Obstrucción de la unión pieloureteral”. 249-272. Ed. McGraw-Hill Interamericana, Mexico D.F., 1998.

9. PARK, J.; BLOOM, D.A.: "Clínicas de Urología de Norteamérica. Obstrucción de la unión pieloureteral”. 177-185. Ed. McGraw-Hill Interamericana, Mexico D.F., 1998.

10. ROSS, J.H.; KAY, R.; KNIPPER, N.S. y cols.: "The absence of crossing vessels in association with ureteropelvic junction obstruction detected by prenatal ultrasonography”. J. Urol., 160: 973, 1998.

11. SCHWALB, D.; LASTARRIA, E.; PARK, T, y cols.: "Massive haematuria from a renal haemangioma with concomitant ureteropelvic junction obstruction". Int. Urol. Nephrol., 25: 121, 1993.
Casos C línicos

Arch. Esp. Urol., 58, 9 (963-965), 2005

\section{DIAGNÓSTICO ECOGRÁFICO DEL VARICOCEE INTRATESTICULAR.}

\author{
Carlos Pascual Mateo, Inmaculada Fernández \\ González, N uria Rodríguez García, Ignacio Romero \\ Cagigal, Gino Espinales Castro y Antonio Berenguer \\ Sánchez.
}

Servicio de Urología. Hospital Universitario de G etafe. $G$ etafe. M adrid. España.

\begin{abstract}
Resumen.- O BJETIVO : El varicocele es una entidad relativamente frecuente; en la gran mayoría de los casos esta dilatación venosa solo afecta a la porción extratesticular, pero en un $2 \%$ de los casos existe afectación intratesticular. Presentamos un caso de varicocele intratesticular izquierdo.
\end{abstract}

MÉTO DO S/ RESULTADO S: Presentamos el caso de un paciente varón de 25 años remitido para estudio por dolor testicular izquierdo y que en su estudio se identificó como único hallazgo un varicocele intratesticular izquierdo.

CON CLUSIO N ES: El varicocele intratesticular es una entidad poco frecuente que puede asociarse a dolor testicular, masa escrotal e infertilidad o bien ser asintomática. Es diagnosticada mediante ecografía Doppler y si es sintomática puede ser manejada mediante ligadura de la vena espermática.

Palabras clave: Varicocele intra testicular. Dolor testicular.

Carlos Pascual Mateo.

Servicio de Urología.

Hospital Universitario de $\mathrm{G}$ etafe.

Carretera de Toledo, Km. 12,500.

$G$ etafe. M adrid. España.

e-mail: carlospa scualmateo @yahoo.es

Trabajo recibido: 17 de marzo 2005 